\title{
Nuevos comportamientos de deshonestidad académica en estudiantes mexicanos: Un estudio exploratorio ${ }^{11}$
}

Félix Javier Reskala Sánchez

PhD. en Psicología

Universidad Nacional Autónoma de México Correo electrónico: felix.reskala@gmail.com
Recibido: 09/05/2019

Evaluado: $18 / 06 / 2019$

Aceptado: 27/08/2019

\section{Abstract}

La deshonestidad académica es un problema grave en las instituciones escolares, ya que daña la reputación de las instituciones educativas, ha sido vinculada a actos deshonestos en los puestos de trabajo y tiene una alta prevalencia. Sin embargo, estudios anteriores se han enfocado en investigar conductas deshonestas ya conocidas por la literatura, y no suelen reportar comportamientos deshonestos nuevos. Por esta razón, este estudio exploratorio cualitativo y cuantitativo buscó identificar nuevos comportamientos deshonestos y reportar la prevalencia del engaño escolar en una muestra de estudiantes mexicanos. Para esto se aplicaron 266 cuestionarios que incluían reactivos tipo Likert y preguntas abiertas. Se encontró una alta prevalencia percibida y 31 comportamientos poco mencionados en la literatura, de los cuales 11 no son mencionados en la literatura revisada. Estos comportamientos incluyen deshonestidad digital y manipulación de los profesores. Se recomienda que futuras investigaciones tomen en cuenta estos nuevos comportamientos deshonestos.

Deshonestidad académica, estudiantes, nuevos comportamientos.

11 Para citar este artículo: Reskala, F. (2020). Nuevos comportamientos de deshonestidad académica en estudiantes mexicanos: Un estudio exploratorio. Informes Psicológicos, 20(2), pp. 155-170 http://dx.doi.org/10.18566/infpsic. v20n2a11 


\title{
New behaviors of academic dishonesty in Mexican students: An exploratory study
}

\begin{abstract}
Academic dishonesty is a serious problem in school institutions, as it damages the reputation of educational institutions. Furthermore, it has been linked to dishonest acts in the workplace and has a high prevalence. However, previous studies have focused on investigating dishonest behaviors already known in the literature, and do not usually report new dishonest behaviors. For this reason, this qualitative and quantitative exploratory study sought to identify new dishonest behaviors and report the prevalence of school cheating in a sample of Mexican students. To do this, 266 questionnaires were applied that included Likert-type items and open-ended questions. A high perceived prevalence and 31 behaviors little mentioned in the literature were found, of which 11 are not mentioned in the reviewed literature. These behaviors include digital dishonesty and teacher manipulation. It is recommended that future research take into account these new dishonest behaviors.
\end{abstract}

Keywords Academic dishonesty, students, new behaviors.

\section{Novos comportamentos de desonestidade acadêmica em estudantes mexicanos: um estudo exploratório}

\section{Resumo}

A desonestidade acadêmica é um problema sério nas instituições escolares, pois prejudica a reputação das instituições educacionais. Esse comportamento tem sido associado a atos desonestos no local de trabalho e tem alta prevalência. No entanto, estudos anteriores se concentraram na investigação de comportamentos desonestos já conhecidos na literatura e, geralmente, não relatam novos comportamentos desonestos. Por esse motivo, este estudo exploratório qualitativo e quantitativo procurou identificar novos comportamentos desonestos e relatar a prevalência de trapaças na escola em uma amostra de estudantes mexicanos. Para isso, foram aplicados 266 questionários que incluíam itens do tipo Likert e perguntas abertas. Foi encontrada alta prevalência percebida e 31 comportamentos pouco mencionados na literatura, dos quais 11 não são mencionados na literatura revisada. Esses comportamentos incluem desonestidade digital e manipulação de professores. Recomenda-se que pesquisas futuras levem em conta esses novos comportamentos desonestos.

\section{Palavras chave}

Desonestidade acadêmica, estudantes, novos comportamentos. 


\section{ntroducción}

La deshonestidad académica se define en esta investigación como una acción intencional que va en contra de los principios éticos de las instituciones educativas y que, además, dan al estudiante una ventaja injusta por encima de sus compañeros, y/o que reducen la exactitud de las inferencias realizadas sobre el desempeño del alumno con base en una prueba o tarea (Cizek, 2004; Diez-Martínez, 2015). Cabe resaltar que a través de este trabajo se usarán los términos "comportamientos engañosos", "conductas deshonestas" y/o "deshonestidad académica" como términos intercambiables para referirnos a la misma acción. Cabe resaltar que la deshonestidad académica es un problema que repercute gravemente en nuestra sociedad (Yekta, Lupton, Takei, Mabudi, \& Jahanfar, 2014), en parte porque se la relaciona con comportamientos de corrupción (Magnus, Polterovich, Danilov \& Savateev, 2002; Vaamonde \& Omar, 2008), actos deshonestos en el trabajo (Nonis \& Owens 2001), y una mala reputación de las instituciones académicas (Sattler, Graeff \& Willen, 2013). Pero, sobre todo, se considera que la alta prevalencia del engaño educativo (Rettinger \& Jordan, 2005), la presión por las calificaciones (Tas \& Tekkaya, 2010) el alto estrés estudiantil (Davis, Grover, Becker, \& McGregor, 1992; Mazo, Londoño, \& Gutiérrez, 2013) y la falta de conciencia de que este tipo de comportamientos son dañinos juega un papel importante sobre la decisión del estudiante de ser deshonesto académicamente (Ling Meng, Othman,
Lawrence D'Silva \& Zohara, 2014). La finalidad de enumerar estos estudios es que sea claro que la deshonestidad académica tiene diversas repercusiones importantes en nuestra sociedad. Aunado a las repercusiones de la deshonestidad académica previamente mencionadas, se considera que ésta tiene una alta prevalencia, ya que diversos estudios mencionan una alta frecuencia de estos comportamientos engañosos que van desde el 28.7\% (Mayhew, Hubbard, Finelli, Harding \& Carpenter, 2009) hasta el 83\% (Cochran, Wood, \& Sellers, 1999) con diversos estudios que reportan prevalencias similares (Avellaneda, 2013; Díaz, Díaz, Díaz \& Franco, 2015; Honz, Kiewra, \& Yang, 2010; Martin, Rao \& Sloan, 2009; Yardley, Domenech, Bates \& Nelson, 2009; Sattler et al., 2013). Si se desea conocer las distintas razones que han dado los estudiantes para ser deshonestos académicamente se recomienda revisar el trabajo de McCabe, Butterfield y Treviñoo (2012). Por lo tanto, hasta ahora se puede observar que deshonestidad académica tiene repercusiones importantes, además de una alta prevalencia.

Sin embargo, a pesar de esta alta prevalencia, la deshonestidad académica no es muy estudiada en México y Latinoamérica. Por ejemplo, Medina y Verdejo (2016) reportan 10 estudios en distintos países de Latinoamérica desde 2004. Siendo más específicos, en México hay más investigaciones, por ejemplo, la realizada por Ayala y Quintanilla (2014) en donde se estudiaron alumnos de una universidad privada y se encontraron altas prevalencias de comportamientos engañosos. Así mismo, se encuentra la realizada por Díaz et al. (2015) en donde se observa que los estudiantes permiten 
copiar respuestas de un examen y aparecer en un trabajo en equipo sin haber colaborado. Diez-Martínez (2014) también llevó a cabo un estudio en donde se reporta la prevalencia de múltiples comportamientos deshonestos. A pesar de que ésta no es una revisión exhaustiva de toda la literatura publicada en México sobre la deshonestidad académica, sí permite observar que la literatura mexicana sobre este tema es escasa. Además, la literatura relacionada con la deshonestidad académica suele enfocarse en investigar comportamientos deshonestos ya conocidos, como el copiar en exámenes y plagiar, pero la mayoría de estudios revisados no investigan nuevos comportamientos engañosos.

Aun así, hay estudios que han preguntado acerca de los comportamientos deshonestos más comunes para los estudiantes, por ejemplo, los trabajos de Castillo y Garybay (2004) y de Canto, Guillermo y Tejada (2011) donde se les preguntó a estudiantes universitarios cuáles comportamientos deshonestos realizaban, llevando a conocer ciertos comportamientos poco comunes. Sin embargo, en la literatura revisada no se han encontrado más estudios que busquen nuevos comportamientos deshonestos en estudiantes universitarios. Por ello, el objetivo de esta investigación es la de reportar cualquier comportamiento engañoso poco mencionado o no reportado previamente en la literatura revisada. De igual manera, debido a que la literatura de deshonestidad académica en Latinoamérica es escasa, este estudio también busca informar sobre la prevalencia de comportamientos deshonestos ya investigados en otras regiones del mundo.

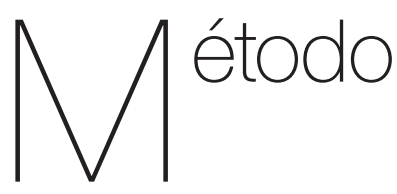

Debido a que la literatura que busca nuevos comportamientos de deshonestidad académica es escasa se realizó un estudio exploratorio con un muestreo transversal no probabilístico por cuota en el cual se tienen datos cuantitativos y cualitativos. La muestra estuvo conformada por 266 estudiantes de licenciatura (132 hombres, 134 mujeres); la edad promedio fue de 20.61 ( $D E=1.684$ ) y pertenecían a 42 carreras, a saber: Arquitectura, Derecho, Física, Ingeniería, Medicina, Química, Pedagogía, Psicología, Sociología, entre otras. Para ser incluido en el estudio, el participante tenía que ser un estudiante de licenciatura de la universidad pública mexicana en la que se realizó el estudio, y aceptar contestar el cuestionario. La prevalencia de la deshonestidad académica fue identificada por medio del cuestionario de Ferrell y Daniel (1995) que usa una escala tipo Likert de siete puntos. Con la finalidad de poder aplicar el cuestionario de Ferrell y Daniel (1995) en una muestra Latinoamericana, se realizó una adaptación al español piloteada con 54 estudiantes de psicología mexicanos (77.4\% mujeres; Edad media $=20.92$, DE $=3.82$ ) elegidos por medio de un muestreo no probabilístico accidental. La retroalimentación de los 54 estudiantes del piloteo permitió que los reactivos fueran más dirigidos, claros, y culturalmente relevantes para los participantes mexicanos. Una vez que se recibió la retroalimentación de los 54 estudiantes se construyó la versión final del instrumento que fue utilizada en 
esta investigación, conformada por 42 reactivos con una escala tipo Likert de siete puntos.

Cabe resaltar que en este cuestionario se preguntó: "¿Con qué frecuencia has observado en otras personas las siguientes conductas?" a diferencia del cuestionario original de Ferrell y Daniel (1995) en donde se pregunta por la prevalencia propia de la deshonestidad académica. Esto se basa en la idea de que los alumnos comúnmente observan a sus compañeros ser deshonestos (Saana, Ablordeppey, Mensah, \& Karikari, 2016), y en que es probable que los estudiantes puedan recordar más acciones deshonestas si toman en cuenta las acciones que han visto hacer a los demás alumnos además de las que ellos mismos han hecho. Los nuevos comportamientos fueron investigados por medio de preguntas abiertas. Después de responder qué tanto habían observado las 42 conductas deshonestas descritas en el cuestionario de frecuencia percibida los participantes leían la definición de deshonestidad académica usada en este proyecto y respondían por escrito las siguientes preguntas: "¿Conoces algunos otros comportamientos de deshonestidad académica?, ¿Cuáles otros? Escríbelos a continuación:". Las respuestas a esta pregunta conformaron los datos cualitativos utilizados en esta investigación.

\section{Procedimiento}

El investigador abordaba a los estudiantes en las áreas comunes de una universidad pública mexicana y preguntaba a los alumnos si podrían contestar un cuestionario aclarando que la participación era anónima y voluntaria. Si los estudiantes accedían a participar se les entregaba el cuestionario de prevalencia percibida que además incluía las preguntas abiertas previamente mencionadas. Una vez que los participantes habían terminado de responder el instrumento se les indicaba la finalidad del estudio y se les agradecía su participación.

\section{Análisis}

Para identificar la prevalencia de cada uno de los 42 comportamientos los datos cuantitativos fueron capturados y analizados en el SPSS 21. La prevalencia se identificó calculando el porcentaje de estudiantes que habían observado, o no, las acciones deshonestas, el puntaje medio, y la desviación estándar de los reactivos de la escala tipo Likert. Asimismo, buscando conocer y mejorar las propiedades psicométricas de la escala, se eliminaron los reactivos que tenían más del 70\% de las respuestas en una sola opción, tenían una asimetría y/o curtosis superiores a dos. De igual manera, se eliminaron aquellos reactivos que no discriminaran entre el 25\% superior e inferior de la muestra, que disminuyeran el Alfa de Cronbach de la escala cuando eran eliminados, y/o que en el análisis factorial presentaran cargas factoriales menores a .40, no cargaran en ningún factor, o que algún factor estuviera conformado por menos de 2 reactivos (procedimientos basados en Fabrigar, Wegener, MacCallum \& Strahan, 1999; Schmitt, 2011; Yong \& Pearce, 2013). Con estos procedimientos se descartaron los reactivos $4,11,17,20$, 21, 22, 24, 30, 32, 36. 
El objetivo del análisis cualitativo era identificar comportamientos de deshonestidad académica no reportados o poco reportados en la literatura revisada por lo que las respuestas cualitativas fueron capturadas usando Microsoft Excel 2007 y analizadas utilizando Atlas ti 7. El análisis cualitativo se basó en las codificaciones abiertas, axiales, y selectivas explicadas por Gibbs (2007) y Boeije (2010). En este análisis, primero se eliminaron las respuestas inválidas o que mencionaran algún comportamiento incluido en el cuestionario de Ferrell y Daniel (1995). Posteriormente, las respuestas que hablaban de comportamientos similares fueron agrupadas, revisadas minuciosamente y comparadas con la definición de deshonestidad académica descrita anteriormente. En específico, para que el comportamiento propuesto por el estudiante fuera considerado deshonestidad académica tenía que cumplir con el primer criterio, y después cumplir con el segundo y/o tercer criterio mencionados a continuación: 1) La conducta tiene que infringir algún principio ético mencionado el código ético de la institución académica. Para esta investigación se utilizó el código ético de la Universidad Nacional Autónoma de México (UNAM, 2015). 2) La acción deshonesta tiene que dar al estudiante una ventaja injusta sobre otros estudiantes; y/o 3) El comportamiento tiene que reducir la calidad de las inferencias sobre el rendimiento del estudiante que estén basadas en el resultado de la tarea en la que se fue deshonesto. Por último, cuando los nuevos comportamientos se identificaron, se buscaron menciones de los mismos en la literatura revisada.

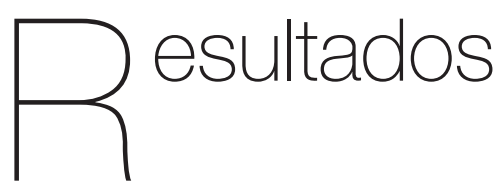

\section{Propiedades psicométricas de la Escala y prevalencia percibida}

En cuanto a los resultados cuantitativos, el instrumento final, eliminando los reactivos mencionados anteriormente, está conformado por 6 factores y un indicador que explican un 53.98\% de la varianza, y que tienen una confiabilidad de .946. Con la finalidad de reportar la prevalencia de los comportamientos deshonestos, en la Tabla 1 se presentan los reactivos que no fueron eliminados por el análisis factorial (o alguno de los procedimientos previos) junto con el porcentaje de estudiantes que han visto esos comportamientos, su media, y su desviación estándar.

Tabla 1.

Prevalencia observada, medias y DE de los comportamientos deshonestos

\begin{tabular}{lccc}
\hline \multicolumn{1}{c}{ Reactivo } & \% lo ha visto & Media & DE \\
\hline $\begin{array}{l}\text { 14. Copiar la tarea de alguien más. } \\
\begin{array}{l}\text { 17. Entregar un reporte basado en el resumen de la } \\
\text { lectura en vez de la versión completa. }\end{array}\end{array}$ 96.20\% & 4.63 & 1.57 \\
\begin{tabular}{l} 
1. Copiar las respuestas de un/a compañero/a en un examen. \\
\hline
\end{tabular}
\end{tabular}




\begin{tabular}{|c|c|c|c|c|}
\hline & Reactivo & $\%$ lo ha visto & Media & $\mathrm{DE}$ \\
\hline 5. & $\begin{array}{l}\text { Pedir las respuestas de un examen a un/a } \\
\text { compañero/a que lo ha realizado anteriormente. }\end{array}$ & $91.40 \%$ & 3.9 & 1.66 \\
\hline 12. & $\begin{array}{l}\text { Que un/a estudiante permita a un/a compañero/a } \\
\text { mirar su examen durante su aplicación. }\end{array}$ & $91.00 \%$ & 3.8 & 1.66 \\
\hline 19. & $\begin{array}{l}\text { Memorizar las preguntas de un examen para } \\
\text { poder revisarlas posteriormente. }\end{array}$ & $88.30 \%$ & 3.8 & 1.78 \\
\hline 6. & $\begin{array}{l}\text { Colocar en la bibliografía referencias sin consultar, para } \\
\text { aparentar un mayor esfuerzo en un trabajo para entregar }\end{array}$ & $86.80 \%$ & 3.54 & 1.65 \\
\hline 2. & Usar un "acordeón" durante la aplicación de un examen. & $86.50 \%$ & 3.42 & 1.59 \\
\hline 37. & Debido a una excusa falsa, retrasar la entrega de un trabajo. & $85.70 \%$ & 3.59 & 1.72 \\
\hline 28. & Permitir a otro/a estudiante copiar respuestas durante un examen & $85.30 \%$ & 3.66 & 1.68 \\
\hline 8. & $\begin{array}{l}\text { Copiar gran parte de un texto sin dar el } \\
\text { crédito correspondiente al autor. }\end{array}$ & $84.20 \%$ & 3.25 & 1.67 \\
\hline 18. & $\begin{array}{l}\text { Memorizar las preguntas de un examen para } \\
\text { poder comunicárselas a otros alumnos. }\end{array}$ & $77.10 \%$ & 3.16 & 1.75 \\
\hline 23. & $\begin{array}{l}\text { Comparar respuestas con un/a compañero/a durante la aplicación } \\
\text { de un examen para conservarlas si ambas son iguales. }\end{array}$ & $77.10 \%$ & 3.24 & 1.82 \\
\hline 16. & Hacer un trabajo escolar para un/a compañero/a de la escuela & $75.90 \%$ & 2.86 & 1.48 \\
\hline 29. & $\begin{array}{l}\text { Intencionalmente copiar frases ajenas a un trabajo } \\
\text { propio sin dar crédito al autor original. }\end{array}$ & $75.60 \%$ & 2.82 & 1.48 \\
\hline 40. & $\begin{array}{l}\text { Permitir que un/a compañero/a resuelva una } \\
\text { tarea, y presentarla como si fuera propia. }\end{array}$ & $74.80 \%$ & 2.87 & 1.55 \\
\hline 25. & $\begin{array}{l}\text { Utilizar un abstract de un artículo científico, como base para } \\
\text { realizar un reporte, en vez de leer el artículo completo. }\end{array}$ & $74.40 \%$ & 2.74 & 1.54 \\
\hline 13. & $\begin{array}{l}\text { Inventar problemas personales para que el/ } \\
\text { la profesor/a coloque una calificación mayor. }\end{array}$ & $72.90 \%$ & 2.89 & 1.69 \\
\hline 33. & $\begin{array}{l}\text { Conversar con un/a profesor/a con la intención } \\
\text { de obtener una mejor calificación. }\end{array}$ & $69.20 \%$ & 2.75 & 1.67 \\
\hline 34. & $\begin{array}{l}\text { En una prueba auto evaluada, corregir las respuestas } \\
\text { incorrectas para mejorar la calificación. }\end{array}$ & $67.30 \%$ & 2.61 & 1.55 \\
\hline 31. & $\begin{array}{l}\text { Colaborar con alguien en un examen en casa, el cual } \\
\text { está pensado para realizarse de manera individual. }\end{array}$ & $66.90 \%$ & 3.09 & 1.98 \\
\hline 35. & $\begin{array}{l}\text { Pedir a un/a compañero/a que escriba un } \\
\text { trabajo, y presentarlo como propio. }\end{array}$ & $64.30 \%$ & 2.38 & 1.41 \\
\hline 7. & Inventar referencias bibliográficas para un trabajo escolar. & $63.90 \%$ & 2.6 & 1.68 \\
\hline 39. & $\begin{array}{l}\text { Hacer cumplidos hacia un/a profesor/a con la } \\
\text { finalidad de obtener una calificación más alta. }\end{array}$ & $60.20 \%$ & 2.5 & 1.64 \\
\hline 27. & $\begin{array}{l}\text { Intercambiar exámenes con un/a compañero/a } \\
\text { mientras éste se está llevando a cabo. }\end{array}$ & $58.60 \%$ & 2.26 & 1.39 \\
\hline 15. & $\begin{array}{l}\text { Visitar a un/a profesor/a después de un examen con } \\
\text { la finalidad de aumentar la calificación asignada. }\end{array}$ & $57.10 \%$ & 2.22 & 1.5 \\
\hline 10. & $\begin{array}{l}\text { Contactar a un/a profesor/a con la intención de } \\
\text { influirlo para que cambie una calificación. }\end{array}$ & $56.40 \%$ & 2.31 & 1.53 \\
\hline 3. & $\begin{array}{l}\text { Estudiar de una copia del examen, la cual fue } \\
\text { obtenida sin la autorización del profesor. }\end{array}$ & $56.00 \%$ & 2.33 & 1.55 \\
\hline
\end{tabular}




\begin{tabular}{|c|c|c|c|}
\hline Reactivo & $\%$ lo ha visto & Media & $\mathrm{DE}$ \\
\hline $\begin{array}{l}\text { 41. Cambiar respuestas de un examen después de } \\
\text { que fue calificado, para entonces reportarle al } \\
\text { instructor que se cometió un error al calificar. }\end{array}$ & $54.90 \%$ & 2.1 & 1.36 \\
\hline $\begin{array}{l}\text { 26. Que un alumno/a "A" le pida a un/a compañero/a } \\
\text { "B" que resuelva el examen en su lugar. }\end{array}$ & $47.40 \%$ & 1.89 & 1.16 \\
\hline $\begin{array}{l}\text { 9. Conseguir una copia de un examen antes de su } \\
\text { aplicación, sin autorización del profesor/a. }\end{array}$ & $46.60 \%$ & 2.03 & 1.45 \\
\hline $\begin{array}{l}\text { 4. Darle regalos a un/a profesor/a para } \\
\text { conseguir una mejor calificación. }\end{array}$ & $43.20 \%$ & 1.78 & 1.25 \\
\hline 42. Coquetear con un/a profesor/a para obtener una mejor calificación. & $42.10 \%$ & 2.07 & 1.57 \\
\hline $\begin{array}{l}\text { 38. Comprar comida a un/a profesor/a con la finalidad } \\
\text { de obtener una mejor calificación. }\end{array}$ & $36.10 \%$ & 1.86 & 1.48 \\
\hline $\begin{array}{l}\text { 24. Tener contacto sexual con un/a profesor/a } \\
\text { a cambio de una calificación. }\end{array}$ & $21.80 \%$ & 1.45 & 1.07 \\
\hline $\begin{array}{l}\text { 32. Insinuarle al profesor/a que habrá contacto } \\
\text { sexual a cambio de una calificación. }\end{array}$ & $21.40 \%$ & 1.45 & 1.11 \\
\hline
\end{tabular}

En la Tabla 2 se presentan los reactivos propuestos por las respuestas cualitativas de los participantes junto con las referencias revisadas en las que se señala alguno de los comportamientos mencionados por los estudiantes o alguno similar. Cabe resaltar que estos reactivos van en contra de uno o más de los principios éticos descritos en el código de ética de la UNAM (2015), además pueden dar una ventaja injusta al estudiante, y/o reducir la exactitud de las inferencias del desempeño del alumno con base en esa tarea.

Tabla 2.

Búsqueda en la literatura revisada por comportamientos similares

Comportamiento
Referencias pasadas que mencionan este comportamiento

1. Indicar al profesor/a que se participó en clase sin haberlo hecho.

2. Evitar decirle al profesor/a que marcó como correcta una respuesta equivocada, esto para conservar una mejor calificación.

3. Dar dinero al profesor/a para cambiar alguna calificación.

Amenazar a un profesor con la finalidad

4. de que mejore la calificación.

5. Ofrecer al profesor presentarle a alguien que le . interesa, a cambio de una mejor calificación.

6. Hacerse amigo del profesor para mejorar la evaluación.

7. Conocer a alguien que tenga acceso a las calificaciones, y que las cambie en favor de algún estudiante.
Ferrell y Daniel, 1995 (primer boceto del instrumento); Alleyne y Phillips (2011); Pratt y McLaughlin, (1989); Vaamonde y Omar (2008)

En un trabajo para entregar, evitar adjuntar el archivo en un correo

8. electrónico, para que el profesor piense que el archivo está ausente por un error, y por lo tanto permita enviar el trabajo más tarde.

Inscribirse en una materia para "apartar lugar" para

9. un compañero/a, y darse de baja de dicha materia cuando el otro alumno/a se desea inscribir. 


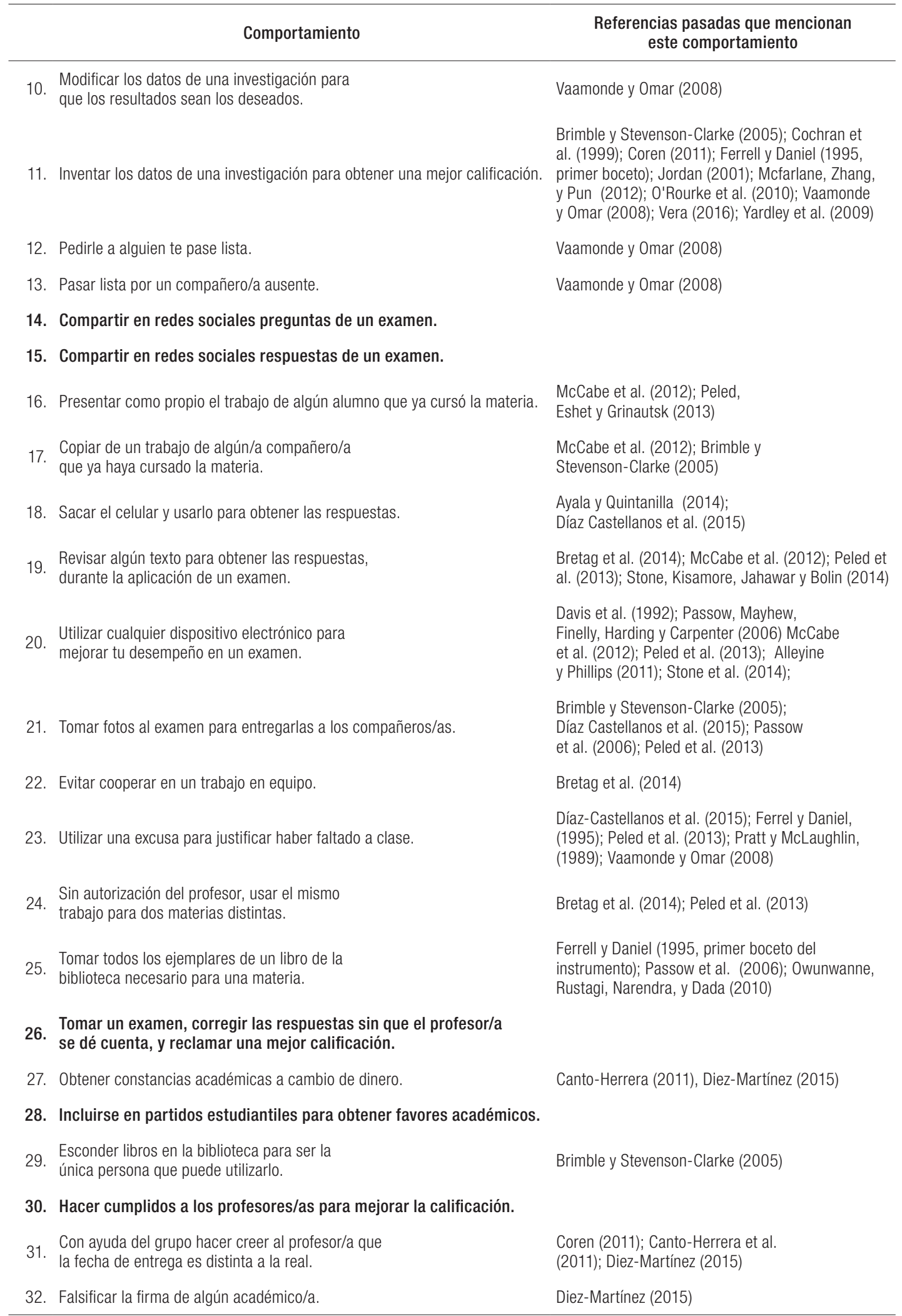

Como podemos observar, 11 de los comportamientos mencionados en este artículo no son nombrados en la literatura revisada. 


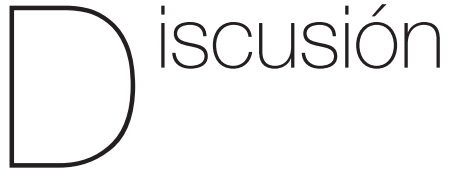

Este estudio tenía la finalidad describir la prevalencia de comportamientos de deshonestidad académica ya conocidos, e identificar nuevos comportamientos engañosos. En los resultados se muestran 32 comportamientos deshonestos de los cuales 11 no fueron reportados por la literatura revisada. Asimismo, más del $90 \%$ de los participantes señalan haber visto a otro estudiante copiar, y más del $80 \%$ reportaron ver algún caso de plagio. En cuanto a las conductas deshonestas cabe resaltar que algunos de estos comportamientos no han sido reportados previamente, como por ejemplo el de "8. Evitar adjuntar el archivo a un correo electrónico para que el profesor crea que el archivo está ausente por error y, por lo tanto, permita enviarlo más tarde". De igual manera, los comportamientos "14. Compartir preguntas de un examen a través de las redes sociales" y "15. Compartir las respuestas de un examen a través de las redes sociales", no han sido citados explícitamente en la literatura revisada. Aunque Brimble y Stevenson-Clarke (2005) y Díaz-Castellanos et al. (2015) indican algo parecido a estas conductas, cuando explican que los estudiantes llegan a usar sus celulares durante los exámenes para ser deshonestos académicamente.

Por lo tanto, es posible que estos comportamientos hayan sido mencionados indirectamente en investigaciones previas, es decir, es muy probable que cuando los estudiantes usan sus teléfonos en el examen, además de buscar en internet las respuestas, estén usando las redes sociales para comunicar y/o conseguir las respuestas de un examen. Por lo tanto, a pesar de que estas acciones no se evidencian explícitamente, es factible que actividades similares o relacionadas hayan sido estudiadas previamente. De un modo similar, la práctica de tomar fotos en el examen y de usar dispositivos electrónicos para obtener las respuestas también son poco citadas en la literatura revisada (con la excepción de Garavalia, Olson, Rusell \& Christensen, 2006). De igual manera, puede ser que la literatura exponga actos deshonestos similares de un modo indirecto, es decir, que no se refieran explícitamente estas conductas en específico pero que se aludan a algunos muy similares; después de todo, de acuerdo con Díaz-Castellanos et al. (2015), los estudiantes utilizan sus teléfonos celulares y el internet para copiar y plagiar. En consecuencia, con base en estos comportamientos, se puede proponer con cierto grado de confianza que algunas de las actividades deshonestas realizadas por los estudiantes sean una indicación de que la tecnología está cambiando los métodos de ser deshonesto académicamente. Por esta razón, se recomienda que futuras investigaciones tomen en cuenta estas nuevas acciones deshonestas "digitales" (McCabe et al., 2012), tanto para aclarar los cambios como para estudiar la prevalencia de las conductas engañosas digitales. Aunque también hay actos deshonestos reportados por los estudiantes que no requieren de la tecnología. Por ejemplo, las conductas de "1. Indicar al profesor/a que se participó en clase sin haberlo hecho", "5. Ofrecer al profesor presentarle a alguien que le interesa, a cambio de una mejor calificación" y "30. Hacer cumplidos a los profesores/as para mejorar la 
calificación" son similares a los comportamientos de manipulación sutil y manipulación fuerte, citados por Ferrell y Daniel (1995), pero no se encontró una mención directa de ellos en la literatura, por lo que es probable que sean poco estudiados y/o nuevos. Aun así, es viable que prácticas similares también hayan sido investigados indirectamente en la literatura, principalmente cuando se habla de manipulación o excusas. Independientemente de la mención, directa o indirecta, de las acciones deshonestas, parece ser que éstas no son estudiadas tan frecuentemente, por lo que se recomienda que sean tomadas en cuenta por futuras investigaciones. También se recomienda que futuros estudios pregunten por los comportamientos "nuevos" de este estudio, ya que si estas acciones tienen una alta prevalencia puede ser que también tengan repercusiones importantes en el entorno académico. De igual manera, caber resaltar que es posible que algunos de las "nuevos" actos reportados en esta investigación puedan ser encontrados en literatura no revisada en esta investigación, ya que, a pesar de realizar una búsqueda bibliográfica importante, es probable que diversas referencias no consultadas incluyan alguna de estas conductas. Sin embargo, incluso si ese es el caso, también es factible que el número de artículos y/o investigaciones que estudian los comportamientos "nuevos" y/o poco investigados de esta investigación no sea muy alto en comparación con otras prácticas deshonestas como el plagio, el cual es estudiado recurrentemente (por ej. Sattler et al., 2013). Debido a esto, se recomienda que se investiguen las conductas deshonestas nuevas de esta investigación y también aquellos que son poco mencionados por la literatura.
De igual manera, los comportamientos encontrados en esta investigación pueden resultar útiles para planear intervenciones que busquen disminuir la prevalencia de la deshonestidad académica. En específico, se recomienda que las intervenciones sean similares a las planteadas por McCabe et al. (2012), las cuales se basan en la promoción de los códigos de honor donde se explique al alumno la integridad y la deshonestidad académica, además de dejar claro que las reglas de ese código tienen que cumplirse y son más que un adorno. Pero, sobretodo, se recomienda usar códigos de honor para permitir crear un ambiente de integridad académica, en donde los alumnos prefieran no ser deshonestos, incluso cuando tienen la oportunidad. Este tipo de intervenciones ha tenido éxito en distintas escuelas angloparlantes (McCabe et al., 2012) y es probable que también tengan éxito en muestras mexicanas.

Otro de los resultados importantes es la alta prevalencia percibida de la deshonestidad académica. Esta alta prevalencia encontrada en los estudiantes de esta universidad debe ser tomada en cuenta por los educadores e investigadores de Latinoamérica, ya que es posible que haya una prevalencia similar en otras universidades mexicanas y/o latinoamericanas. Estos datos son similares a la prevalencia de auto reporte de estudios previos (por ej: Honz et al., 2010) y, por lo tanto, es factible que lleve a desconfianza en las instituciones académicas (Sattler et al., 2013), comportamientos laborales deshonestos (Nonis \& Owens, 2001) y corrupción en general (Magnus et al., 2002). Asimismo, la relación entre la deshonestidad académica y la corrupción en general puede ser de particular interés para México, un país con un problema de 
corrupción importante (Ayala \& Quintanilla, 2014). Por último, cabe resaltar que la definición de Deshonestidad Académica usada en este proyecto puede resultar de utilidad para futuras investigaciones, independiente del método y/o del lugar donde se haga el estudio.

A pesar de los resultados, este estudio tiene diversas limitaciones. Primero, la muestra es no probabilística, por lo tanto, es muy probable que los resultados no sean generalizables a todos los estudiantes mexicanos o a estudiantes en otros países. De igual manera, la prevalencia reportada se basa en cuantos actos deshonestos habían visto los participantes, por lo que es posible que sea mayor que la real. Además, aunque los participantes mencionaron prácticas deshonestas nuevas y/o poco investigadas, es posible que haya nuevos comportamientos que no hayan sido reportados en este estudio. A pesar de estas limitaciones, los resultados proveen información de prevalencia percibida de estudiantes mexicanos, en los cuáles la literatura es escasa (véase Medina \& Verdejo, 2016; para una revisión de la literatura latinoamericana). La deshonestidad académica es prevalente en la universidad mexicana en la que se realizó el estudio, y, al parecer, las frecuentes conductas engañosas están cambiando con el tiempo. Además, parece que están surgiendo nuevos comportamientos de engaño escolar y otros están dejando de realizarse, o los medios para hacerlos están cambiando. Por lo tanto, se recomienda que futuras investigaciones describan la prevalencia de los 31 comportamientos deshonestos, poniendo énfasis en las 11 conductas no mencionadas en la literatura revisada, en alumnos de licenciatura y de otros niveles educativos y/o contextos.
Los resultados de esta investigación, y de las futuras investigaciones que estudien conductas deshonestas nuevas o poco estudiadas, nos pueden ayudar a entender cómo y qué tanto los estudiantes son deshonestos, y si se tiene esta información es más probable que en el futuro se puedan desarrollar mejores intervenciones para disminuir la deshonestidad.

\section{Agradecimientos}

Se agradece al Consejo Nacional de Ciencia y Tecnología por el financiamiento de este proyecto. Número de Becario 582627, CVU 698179.

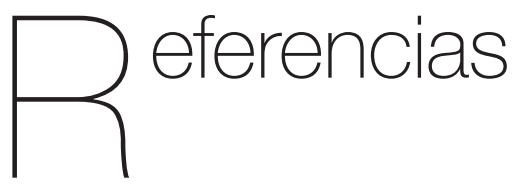

Alleyne, P., \& Phillips, K. (2011). Exploring academic dishonesty among university students in Barbados: An extension to the Theory of Planned Behavior. Journal of Academic Ethics, 9(4), 323-338. doi: 10.1007/s10805-011-9144-1

Avellaneda, S. J. (2009). Tolerancia de los estudiantes de pregrado ante los comportamientos desviados en el aula de clase: Un estudio comparativo. Activos, 11(20), 6-32. https://doi.org/10.15332/ s0124-5805.2013.0020.01

Ayala, G., E. A., \& Quintanilla, D. C. M. (2014). Attitudes and causes of cheating among Mexican college students: An exploratory research. Magis, Revista Internacional de Investigación en Educación, 6(13), 17-30. 
Saana, S. B., Ablordeppey, E., Mensah, N. J., \& Karikari, T. K. (2016). Academic dishonesty in higher education: students' perceptions and involvement in an African institution. BMC Research Notes, 9, 234-247. http:// doi.org/10.1186/s13104-016-2044-0

Boeije, H. (2010). Analysis in Qualitative Research. Great Britain: Sage Publications.

Bretag, T., Saadia, M., Wallace, M., Walker, R., McGowan, U., East, J., Green, M., Partridge, L., \& James, C., (2014). 'Teach us how to do it properly!' An Australian academic integrity student survey. Studies in Higher Education, 39(7), 1150-1169, doi: 10.1080/03075079.2013.777406

Brimble, M., \& Stevenson-Clarke, P. (2005). Perceptions of the prevalence and seriousness of academic dishonesty in Australian universities. The Australian Educational Researcher, 32(3), 19-44. doi: 10.1007/bf03216825

Canto, H., P.J., Guillermo, G. M. C. \& Tejada, L., M. A. (2011). Opinión de estudiantes de posgrado acerca de las conductas no éticas cometidas por sus compañeros. En Hirsch Adler y López Zavala (Coords.), Ética y Valores Profesionales: Trece experiencias de investigación universitaria en México (pp. 83-112). México: Editorial UAS

Castillo, M., A. \& Garibay, P. (2004). Ética en la investigación científica y la educación superior: Perspectiva de una estudiante de licenciatura. En Aluja \& Birke (Coords.), El papel de la ética en la investigación científica (pp. 181-202). México: Fondo de Cultura Económica.

Cizek, G. J. (2004). Cheating in academics. In C. Spielberger (Ed.), Encyclopedia of applied psychology (pp. 307-311). San Diego, CA: Academic Press.

Cochran, J., Wood, P., \& Sellers, C. (1999). Shame, embarrassment, and formal sanction threats: extending the deterrence/ rational choice model to academic dishonesty. Sociological Inquiry, 69(1), 91-105.

Coren, A. (2011). Turning a blind eye: Faculty who ignore student cheating. Journal of Academic Ethics, 9(4), 291-305.

Davis, S., Grover, C., Becker, A., \& McGregor, L. (1992). Academic dishonesty: Prevalence, determinants, techniques, and punishments. Teaching of Psychology, 19(1), 16-20.

Díaz, E.E., Díaz, C. Díaz, K., \& Franco, M. (2015). Ética: ¿Cuál es la percepción de los jóvenes universitarios? Revista de Estudios y Experiencias en Educación, 14(27), 137-146.

Diez-Martínez, E. (2015). Deshonestidad académica de alumnos y profesores: Su contribución en la desvinculación moral y corrupción social. Sinéctica, (44), 1-17. Recuperado el 08 de abril de 2016 de http:// www.scielo.org.mx/scielo.php?script=sci_ arttext\&pid=S1665109X2015000100014\& | ng=es\&tlng=es.

Fabrigar, L., Wegener, D., MacCallum, R., \& Strahan, E. (1999). Evaluating the use of exploratory factor analysis in psychological research. Psychological Methods, 4(3), 272-299. http://dx.doi. org/10.1037//1082-989x.4.3.272

Ferrell, C. \& Daniel, L. (1995). A frame of reference for understanding behaviors related to the academic misconduct 
of undergraduate teacher education students. Research in Higher Education, 36(3), 345-375.

Garavalia, L., Olson, E., Russell, E., \& Christensen, L. (2006). How do students cheat? In Anderman \& Murdock (Eds.) Psychology of Academic Cheating. (pp. 33-55). USA: AcademicPress. https://doi. org/10.1016/B978-0-12-372541-7.X5000-1

Gibbs, G. (2007). Analyzing Qualitative Data. Great Britain: Sage Publications.

Honz, K., Kiewra, K., \& Yang, Y.S. (2010). Cheating perceptions and prevalence across academic settings. Mid-Western Educational Researcher, 23(2), 10-17.

Ling Meng, C., Othman, J., Lawrence D'Silva, J. \& Zoharah, O. (2014). Ethical decision making in academic dishonesty with application of modified Theory of Planned Behavior: A review. International Education Studies, 7(3), 126-139.

Jordan, A., (2001) College student cheating: The role of motivation, perceived norms, attitudes, and knowledge of institutional policy. Ethics \& Behavior, 11(3), 233-247. DOl: 10.1207/S15327019EB1103_3

Magnus, J. R, Polterovich, V.M., Danilov D. L. \& Savvateev. A. V. (2002) Tolerance of cheating: an analysis across countries. The Journal of Economic Education, 33(2), 125 135. doi: 10.1080/00220480209596462.

Mayhew, M., Hubbard, M. S., Finelli, C., Harding, T. \& Carpenter, D. (2009). Using structural equation modeling to validate the Theory of Planned Behavior as a model for predicting student cheating. The Review of Higher Education, 32(4), 441-468.
Martin, D., Rao, A. \& Sloan, L. (2009). Plagiarism, integrity and workplace deviance: a criterion study. Ethics \& Behavior, 19(1), 36-50. doi: 10.1080/10508420802623666

Macfarlane, B., Zhang, J., \& Pun, A. (2012). Academic integrity: a review of the literature. Studies in Higher Education, 39(2), 339358. doi: 10.1080/03075079.2012.709495

Mazo, R., Londoño, K., \& Gutiérrez, Y, F. (2013). Estrés académico en estudiantes universitarios. Informes Psicológicos, 13(2), 121-134.

McCabe, D., Butterfield, K. \& Treviño, L. (2012). Cheating in college: Why students do it and what educators can do about it. Baltimore: Johns Hopkins University Press.

Medina, M. \& Verdejo, A. (2016). Una mirada a la deshonestidad académica y el plagio estudiantil en algunas universidades de siete países de América Latina. Trabajo presentado en Seminario: La situación de la Educación Superior Virtual en América y el Caribe. Recuperado el 25 de octubre de 2016 de http://reposital.cuaed.unam. mx:8080/jspui/handle/123456789/4673

Nonis, S. \& Owens, C. (2001). An examination of the relationship between academic dishonesty and workplace dishonesty: a multicampus investigation. Journal of Education for Business, 77(2), 69-77. doi: 10.1080/08832320109599052

O'Rourke, J., Barnes, J., Deaton, A., Fulks, K., Ryan, K., \& Rettinger, D. (2010). Imitation is the sincerest form of cheating: the influence of direct knowledge and attitudes on academic dishonesty. Ethics \& Behavior, 20(1), 47-64. doi: 10.1080/10508420903482616. 
Owunwanne, D., Rustagi, Narendra, R., \& Dada, R., (2010). Student's perceptions of cheating and plagiarism in higher institutions. Journal of College Teaching \& Learning, 7(11), 59-68. doi: 10.19030/tlc. v7i11.253

Passow, H., Mayhew, M., Finelly, C., Harding, T., \& Carpenter, D. (2006). Factors influencing engineering student's decisions to cheat by type of assessment. Research in Higher Education, 47(6), 643-684. doi: 10.1007/ s11162-006-9010-y

Peled, Y., Eshet, Y. \& Grinautski, K. (2013). Perceptions regarding the seriousness of academic dishonesty amongst students - A comparison between face-to-face and online courses. Proceedings of the Chais conference on instructional technologies research 2013: Learning in the technological era, Raanana, Israel. Retrieved from https://www.researchgate. net/publication/250305863_Perceptions_ Regarding_the_Seriousness_of_ Academic_Dishonesty_amongst_ Students_-_A_comparison_Between_ Face-to-Face_and_Online_Courses

Pratt, C. \& McLaughlin, G. (1989). An analysis of predictors of college student's ethical inclinations. Research in Higher Education, 30(2), 195-219.

Rettinger, D. A., \& Jordan, A. E., (2005). The relations among religion, motivation, and college cheating: a natural experiment. Ethics \& Behavior, 15(2), 107-129. doi: 10.1207/s15327019eb1502_2

Sattler, S., Graeff, P., \& Willen, S. (2013) Explaining the decision to plagiarize: an empirical test of the interplay between rationality, norms, and opportunity.
Deviant Behavior, 346, 444-463. doi: 10.1080/01639625.2012.735909.

Schmitt, T. (2011). Current methodological considerations in exploratory and confirmatory factor analysis. Journal of Psychoeducational Assessment, 29(4), 304-321. http://dx.doi. org/10.1177/0734282911406653.

Stone, T., Kisamore, J., Jawahar, I., \& Bolin, J. (2014). Making our Measures Match Perceptions: Do Severity and Type Matter When Assessing Academic Misconduct Offenses?. Journal of Academic Ethics, 12(4), 251-270. doi: 10.1007/ s10805-014-9216-0.

Tas, Y. \& Tekkaya, C. (2010). Personal and contextual factors associated with students' cheating in science. The Journal of Experimental Education, 78(4), 440-463. doi: 10.1080/00220970903548046

Universidad Nacional Autónoma de México (2015). Código de ética de la universidad nacional autónoma de México. Recuperado de http://dgapa.unam.mx/ images/etica/2015_codigo-etica-unam.pdf el 25/04/2017.

Vaamonde, J.D. \& Omar, A., (2008). La deshonestidad académica como un constructo multidimensional. Revista Latinoaméricana de Estudios Educativos, 38(3-4), 7-27.

Vera, H. (2016). El plagio y la autonomía de las instituciones académicas. Perfiles Educativos, 38(154), 28-34.

Yardley, J., Domenech, M., Bates, S. \& Nelson, J. (2009). True confessions? Alumni's retrospective reports on undergraduate 
cheating behaviors. Ethics \& Behavior, 19(1), 1-14. doi: 10.1080/10508420802487096.

Yekta, S.A., Lupton, R., Takei, T., Mabudi, K., \& Jahanfar, M. (2013). A comparative study of attitudes of academic staffs towards academic dishonesty between USA and Iranian higher educational institutions. International Research in Education, 2(1), 41-50. doi: 10.5296/ire.v2i1.4178
Yong, A., \& Pearce, S. (2013). A beginner's guide to factor analysis: focusing on exploratory factor analysis. Tutorials in Quantitative Methods for Psychology, 9(2), 79-94. doi: 10.20982/tqmp.09.2.p079 